\title{
Electrooculographic indices of changes in simulated flying performance
}

\author{
TERRY L. MORRIS \\ Texas A\&M University, College Station, Texas
}

\begin{abstract}
Recent investigations have revealed statistically reliable relationships between signal detection by human subjects performing vigilance tasks and changes in the electrophysiologically recorded waveform of the eyeblink. Thus, this relatively simple and easy-to-obtain measure may have potential use as a predictor of impaired performance. This report describes a study in which eyeblink data were recorded on analog tape while subjects (instrument-rated pilots) "flew" a computer-generated flight plan in a General Aviation Trainer (GAT-1). Eyeblink data were reduced and scored with an adaptation of software from the Washington University Behavioral Research Laboratory. Subjects' "flying" performance was evaluated with the Pilot Performance Evaluating System (PPES) implemented on a Digital Equipment Corporation (DEC), PDP-11/34 computer. The PPES samples each of four primary flight instruments once per second. It scores performance by generating a combined error score for each maneuver it assigns on the flight-plan display in the cockpit. The PPES/GAT-1 linkup has proved to be a valuable research tool with which to realistically evaluate stress effects and physiological correlates of flying performance in a laboratory setting. This study examined the relationships between eyeblink and flying performance scores collected during a pilot-fatigue study. Analysis of the data supports the use of computerscored eye movement measures as predictors of flying performance decrements.
\end{abstract}

Recent aircraft technology has resulted in the development of aircraft with greatly extended range and flight duration capabilities as well as highly increased performance. However, the positive factor of increased system performance may also have a negative aspect, since increased aircraft performance increases the physical and mental demands on the pilot and, predictably, pilot fatigue. Increases in flight durations and the complexity of operating a highly sophisticated aircraft have made the problems of pilot fatigue and the associated performance decrement an issue of growing concern.

It is one of the goals of human factors research to investigate the mechanisms by which fatigue affects pilot performance. Understanding this relationship will aid human factors engineers in accomplishing their goal of improving the design of aircraft and other human-machine systems to better allow for the capabilities and limitations of humans within those systems. This effort also requires that systems be created with which to accomplish the scientific research necesary to collect appropriate data.

There is a long-recognized need for measures sensitive to fatigue and its effect on "flying-related performance" (defined here as the multidimensional tracking task associated with flying an aircraft). As pilots approach the time limits of endurance, their overall performance capacity, diminished by fatigue, may go largely undetected

The author's current mailing address is: U.S. Air Force School of Aerospace Medicine/VN, Brooks Air Force Base, TX 78235. until acceptable performance momentarily ceases. Also, different kinds of performance such as manual control, decision making, long-term memory, short-term memory, etc., are differentially susceptible to fatigue.

Many researchers have examined the usefulness of certain physiological changes as correlates of noticeable performance decrements attributed to fatigue. As Jahns (1973) pointed out, "a real need exists to standardize and improve physiological techniques so that meaningful criteria for crew systems design can be derived from their application"' (p. 34).

Although some research has been carried out on the relationships among electrooculographic (EOG) measurements, increased fatigue, and variability in performance, little has been done to examine the relationships among the EOG, performance related to flying, and the effects of fatigue and time on task.

This report describes the development and testing of a system that allows the use of a flight simulator as a research tool. A system was needed with which to examine pilots' physiological variables and the relationship of these variables to changes in flying performance. To evaluate the usefulness of this system, specific eye movements, measured with the electrooculogram (EOG), were examined as trained instrument pilots "flew" sorties in a flight simulator. On the basis of the literature, the specific predictions were that eyeblink rate and duration, long eye closure rates, and saccade velocities would be positively correlated with performance decrements: eyeblink amplitude and saccade frequency would be negatively correlated with performance decrements. 


\section{METHOD}

\section{Subjects}

Of the 10 pilots evaluated, 5 were currently active Air Force pilots, 4 more were on active duty but were no longer on flying status, and 1 was a former Air Force pilot employed by the Air Force as a civilian researcher.

Of the 10 subjects, 9 were men and 1 was a woman. Their ages ranged from 25 to 42 years (mean $=36.1$ years). Their flight experience in total hours logged ranged from 750 to 3,500 (mean $=2,115 \mathrm{~h}$ ), and ranged in years of flying from 3 to 21 (mean $=12.7$ years).

\section{Apparatus}

The organization of the components in the system is shown in Figure 1.

The GAT-1 moving-base flight simulator (Model B633000, manufactured by the Link Division, Singer-General Precision, Inc.) used in this study was adapted as a performance-estimating device. The GAT-1 is a basic instrument-flight trainer with cockpit motion and engine sound simulation. It provides the pilot with standard aircraft cockpit instrumentation, including pitch and roll attitude, altitude, airspeed, heading, turn rate, turn coordination, and vertical velocity. Using standard aircraft controls, the pilots were able to simulate flight on instruments, with the exception that no navigation simulation was provided. Engine sound simulation was adjusted to $80 \mathrm{dBA}$ with the engine running at 2,000 rpm, altitude zero, and motion off. An intercom was installed to allow the pilots to communicate with the experimenter, who was stationed outside the cockpit.

At the U.S. Air Force (USAF) School of Aerospace Medicine, the GAT-1 has been used for numerous studies to evaluate the effect' on pilot performance of various chemical and environmental stressors (Henry, Davis, Engelken, Triebwasser, \& Lancaster, 1974; Henry, Flueck, Sanford, Keiser, McNee, Walter, Webster, Hartman \& Lancaster, 1974; Henry, Turner, \& Matthie, 1974). Perelli (1980) adapted the GAT-1 to a system that linked it to a computer. The computer software developed for this system (Threatt \& Perelli, 1977) allowed the researcher to input a series of different, but equivalent, flight plans of any desired length or complexity, which the computer would then present step by step to the pilots as they flew the simulators. The earlier system presented each flight segment separately, allowing pilots to relax between segments (and to recover to some extent from fatigue effects). The later, computer-generated, flight plans were continuous, and pilots were scored virtually throughout the flight.

Flight maneuver instructions to the pilot were displayed on a 13-in. (diagonal measure) video (CRT) screen (Conrac Data Monitor, Model 7211RS13, Conrac, Inc., Covina, $\mathrm{CA}$ ). With the simulator windows covered and the cockpit door closed, the CRT and instrument lamps were the only sources of illumination inside the cockpit. The CRT intensity control was adjusted to give the "sharpest" characters.

Flight maneuver instructions to the pilots, as well as the collection of flight performance data from the GAT1 , were controlled by means of a computer program implemented on a Digital Equipment Corporation (DEC) PDP-11/34 computer system. This program, called the Pilot Performance Evaluation System (PPES), was created to provide an integrated system linking the GAT-1 to the computer (Threatt \& Perelli, 1977). It allows evaluation of output signals from flight instruments in the cockpit as these signals vary in relation to parameters prescribed by the program. For example, as the pilot deviates from

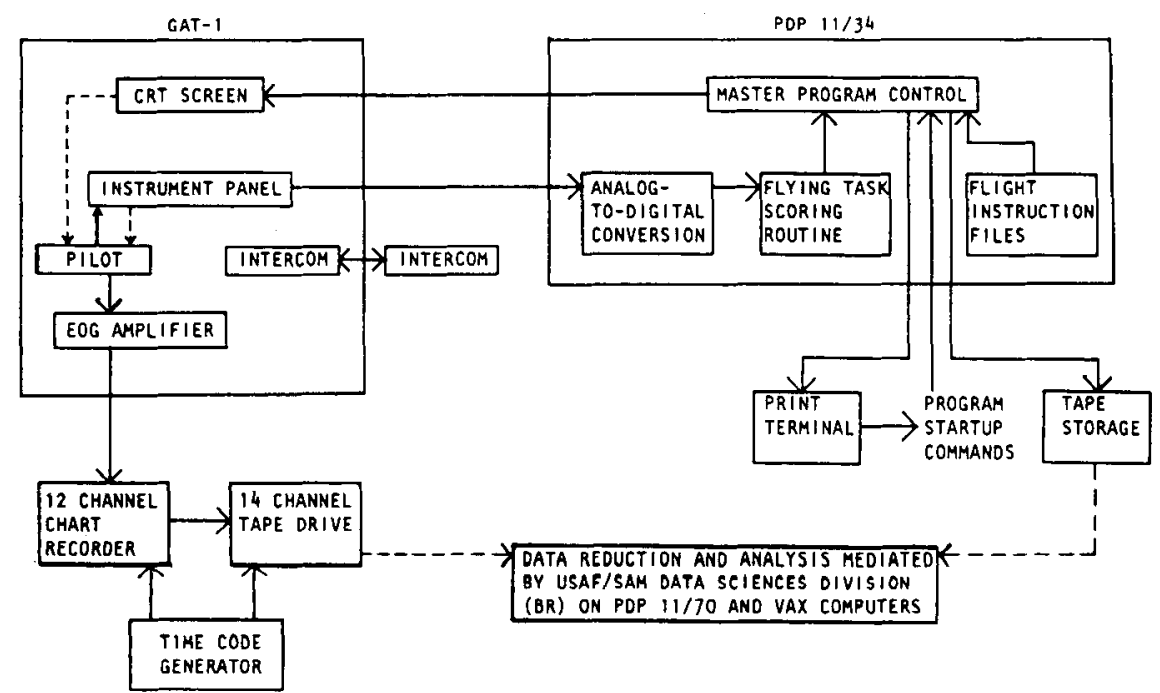

Figure 1. Linked components of the GAT-1, computer, and physiological measurement recording system. 
the heading displayed on the CRT, the time outside the prescribed range of values is scored as error.

The study described here concerned itself, in part, with investigating the relationships of certain components of eye movement to performance changes due to fatigue. In order for a measurement of eye movement or any other physiological variable in the human to be a useful tool in evaluating stress effects in operational flying, certain requirements must be met. First, the measurement technique must be easily and quickly instrumented. Second, it must be sensitive to the physiological variable of interest without being so sensitive that "noise" will obscure the useful data. Third, it must be relatively "invisible" to the pilot so that it does not interfere with or change flying performance. One such measure of eye movement is the EOG.

EOG data obtained during the experiment were acquired by attaching Beckman silver/silver-chloride electrodes to the subjects. This type of electrode was used because of its ionic stability and suitability for high amplifier input impedance, direct current recording (Oster \& Stern, 1980). These electrodes were filled with an electrode cream (EC-2, Grass Instruments Co.) and attached to the skin with adhesive rings. The EOG signals from the electrodes were amplified differentially, with a bandpass of 0.05 to $50 \mathrm{~Hz}(6 \mathrm{~dB}$ down) by physiological preamplifiers (Model 2123, Data, Inc., Fort Collins, CO), and then output to the PDP-11/34 for storage on a Honeywell 101 14-channel tape deck with real-time record monitor and IRIG-B time code. Real-time monitoring was afforded by write-out on a Beckman R-511 4-channel polygraph.

\section{Flight Plans}

The sortie to be flown by each pilot lasted approximately $4.5 \mathrm{~h}$ (Figure 2). Each sortie began with a preparatory command, continued with a takeoff command followed by the scored portion of the "flight," and ended with a

\begin{tabular}{|c|c|c|c|c|c|c|c|c|}
\hline & PF & \multicolumn{2}{|c|}{$T / 0$} & & & & & \\
\hline $\mathrm{CA}$ & \multicolumn{2}{|c|}{ FNT 1} & $\mathrm{CA}$ & SLT I & CA & FNT 2 & $C A$ & SLT 2 \\
\hline $\mathrm{CA}$ & \multicolumn{2}{|c|}{ FMT 3} & $\mathrm{CA}$ & SLI 3 & $\mathrm{CA}$ & FMT 4 & $C A$ & SLT 4 \\
\hline $\mathrm{CA}$ & \multicolumn{2}{|c|}{ FMT 5} & CA & SLT 5 & CA & FMT 6 & $\mathrm{CA}$ & SLT 6 \\
\hline CA & \multicolumn{2}{|c|}{ FMT 7} & $\mathrm{CA}$ & SLT 7 & $\mathrm{CA}$ & FMT 8 & CA & SLT 8 \\
\hline & & SDC & & & & & & \\
\hline
\end{tabular}

$\mathrm{CA}=$ course adjustment $1 \mathrm{~min} ; \mathrm{FMT}=$ flight maneuver task: 16 min; L = landing: 12 min; PF = preflight: 2 min; SDC = subjective fatigue and SSS data collection: $6 \mathrm{~min}$; SLT = straight and level flight task: $10 \mathrm{~min} ; \mathrm{T} / \mathrm{O}=$ takeoff: 12 min. *Includes Sleep Survey Card.

Figure 2. Sequence of events for the 4.5-h flight plan. landing. The scored portion consisted of eight legs, with each leg made up of two segments:

(1) Flight Maneuvers Task (FMT)-This segment consisted of a series of eight different maneuvers, including climbs, descents, turns, and combinations of each of these. Each of these segments lasted $16 \mathrm{~min}$.

(2) Straight and Level Task (SLT)-This segment required the pilot to fly straight ahead for $10 \mathrm{~min}$ at fixed altitude, heading, and airspeed.

The computer displayed flight instructions to the pilots on the cockpit CRT. Each maneuver consisted of preparatory instructions, followed by an execution command informing them which maneuver to perform. A countdown number in the lower right corner of the CRT screen indicated how much time remained to begin, and then to complete, each maneuver (5-sec increments). Flight maneuvers within each different FMT were assigned by Latin square and were selected from eight maneuvers counterbalanced for climbs, descents, left turns, and right turns. There were eight possible FMTs; these were assigned, also by Latin square, to eight different flight plans. These 16-segment flight plans (plus takeoffs and landings) were adapted from the original flight plans used by Perelli (1980). The revised version, used here, allowed additional data points to be collected for the FMT performance scores. This in turn allowed a more statistically reliable analysis of the relationship between performance scores and EOG data.

\section{Performance Measurements}

For the purposes of this study, "performance measurement" was limited to considerations involving the evaluation of "flying-related performance," defined as the multidimensional tracking task of maneuvering an aircraft in flight or an aircraft simulator in simulated flight. Scientists and engineers have concerned themselves for some time with the need for techniques with which to measure and evaluate changes in a pilot's performance in flight as the pilot is subjected to varying types and degrees of stressful situations. Not the least of these "situations" is pilot fatigue. Flying performance changes due to fatigue are subtle, and measures of them, accordingly, must be quite sensitive. The effects of fatigue on pilot performance are intermittent in the early stages. There are momentary lapses of attention that increase performance variability, but do not appear to affect the overall success of performance until a critical signal is missed or fatigue becomes extreme and satisfactory performance ceases.

Part of the problem in pilot performance measurement has been the use of the dichotomy of "satisfactory/unsatisfactory." As increasing fatigue requires the pilot to call on reserves of energy to continue flying, performance remains "satisfactory" until an error of omissin or other mistake results in "unsatisfactory" performance. A measure of pilot performance based on this construct will not fill the need to identify that period when a pilot is still flying safely, but will soon not be. "Window scoring" methods that are based on time on target 
or time off target appear on the surface to be continuous measures, but in fact are only repeated measures of the dichotomy "satisfactory/unsatisfactory." These "window" methods also suffer from a lack of definition for window width. Whereas one study may use a window of $\pm 10 \mathrm{ft}$ of altitude, another might use $\pm 100 \mathrm{ft}$. It is obvious that a pilot who starts a flight varying at $\pm 15 \mathrm{ft}$ and ends it at $\pm 90 \mathrm{ft}$ due to fatigue effects will produce anomalous scores using the two "window" criteria just mentioned.

The concept of "variability versus accuracy" implies the measurement of mean performance lying within predetermined limits versus the measurement of variability of performance. It is apparent from the literature that variability is generally better suited than accuracy to error measurement in continuous tasks such as flying and driving. Obviously, variability may increase over time with no change in the mean (accuracy), and the literature indicates that significant changes in mean performance values due to fatigue will typically occur only at a point in time at which it is too late to prevent catastrophic failures in performance. Variability in performance is, at least, the most useful measure for evaluating the early stages of fatigue.

However, performance accuracy cannot be ignored. The problem encountered in earlier evaluations of tracking performance tasks, such as flying, was not in using measures of accuracy, but in using accuracy as the only measure. Conversely, the fallacy of using variability alone can be demonstrated if one considers two pilots separately flying the same cross-country course. Assume that both pilots continuously vary at $\pm 2^{\circ}$ about their mean heading for the entire flight, and that one pilot is varying about the assigned course and the other about a mean that is $10^{\circ}$ off course. In $1 \mathrm{~h}$ at $200 \mathrm{mph}$, one pilot will arrive at the destination and the other will miss it by 35 miles.

These considerations suggest that the measurement of performance in flying-related tasks should include the variability of tracking (such as standard deviation), weighted by accuracy (deviation of the mean from the assigned value). Such a measure was used here. In this study, pilot performance was measured and scored indirectly as the output of system performance. In this case, the system performance measures were those acquired from the flight instrumentation on the cockpit instrument panel of the GAT-1. The following four instruments were scored for error both in terms of "variability" (mean and standard deviation) and in terms of the total number of seconds outside the "window" indicated:

(1) Airspeed: $\pm 5 \mathrm{mph}$

(2) Heading: $\pm 2.5^{\circ}$

(3) Altitude: $\pm 40 \mathrm{ft}$

(4) Vertical velocity: $\pm 150 \mathrm{ft} / \mathrm{min}(150 \mathrm{fpm})$.

These values were chosen because of their correspondence to the Perelli (1980) study. The corresponding criteria for the FAA Pilot Examination (FAA Flight Test Guide AC61-17B) are slightly more strict for vertical velocity $\left( \pm 100 \mathrm{fpm}\right.$ ) and slightly less strict for heading $\left( \pm 5^{\circ}\right)$ and altitude $( \pm 50 \mathrm{ft})$.

All four instruments were scored for the SLT. During each maneuver of the FMT, only those instruments that were to be held constant were scored. The flight instruments were not sampled during takeoffs and landings.

\section{Procedure}

The subjects were instructed to be present at the USAFSAM/VNB Psychophysiology Function (Building 170, Brooks AFB) for two daytime 1 -h training sessions in the GAT-1 simulator. During the second session, subjects were connected to EOG-measuring equipment in order for them to become accustomed to wearing the electrodes. Also, due to individual differences in subjects' EOG baseline potential and signal amplitudes, this was done to allow for preadjustment of the EOG measuring equipment. Each training session was conducted in the morning on separate days. Additionally, each subject's practice sessions were on days other than his or her experimental sortie.

On the day of the sortie, subjects reported to their own work environment or to the simulator laboratory at $0100 \mathrm{~h}$ and were monitored by the investigator or his representative to ensure that they remained awake until the end of the actual simulator "flight," which lasted from 1300 until $1730 \mathrm{~h}$. Subjects were encouraged to bring along sedentary tasks that would serve to occupy them and aid them in remaining awake during the $12 \mathrm{~h}$ preceding the 4.5-h "flight." The purpose of alerting the subjects at such an early hour was based on the findings of previous studies in which subjects had not reached desired levels of "fatigue" during a 4-h flight unless they were somewhat fatigued at the start (Mutto \& Wierwille, 1982; Stave, 1977). Subjects were prefatigued to produce errors, not to examine any specific work schedule.

Prior to entering the GAT-1, the subjects spent approximately $.5 \mathrm{~h}$ being instrumented with the EOG electrodes. Electrode placement followed the method described in related studies (Beideman \& Stern, 1977; Oster \& Stern, 1980). Vertical EOB (VEOG) was measured from electrodes placed on the skin above and below, and directly in line with, the centered right pupil. Horizontal EOG (HEOG) was measured from electrodes placed near the outer canthus of each eye and in line with the centered pupils. A fifth (ground) electrode was placed in the center of the forehead (midline, between the frontal eminences).

Subjects were then seated in the cockpit so that the EOG electrodes could be connected to the preamplifiers. Following an equipment check and alignment, subjects "flew" the 4.5-h sortie.

Subjects were required to fly the sortie without a rest break, since it had been demonstrated previously that vigilance performance and the performance of similar tasks such as the tracking required here can be maintained effectively in spite of fatigue when subjects are given short rest breaks (Bergum \& Lehr, 1962; Colquhoun, 1959). 
Sussman (1970) demonstrated that a 4-min rest break at the end of $4 \mathrm{~h}$ of work resulted in almost complete performance recovery in a tracking task.

\section{Data Reduction}

Performance data were deposited in a PDP-11/34 computer file on disk, collected during the experiment. The computer recorded the simulated airspeed, altitude, heading, and vertical velocity once per second for each scored maneuver. The resulting file consisted of 960 samples of each of the four flight parameters for each of the eight flight maneuver tasks and 600 samples of each of the four flight parameters for each of the eight straight and level tasks. These data files were transferred to digital tape and entered into the VAX 11/780 computer at the USAFSAM Data Sciences Division (BR). The data file was then processed with a FORTRAN program that reduced the data to means and standard deviations of each of the four instrument parameters for each of the 16 scored maneuver tasks. The program then computed error scores for each maneuver representing altitude error, airspeed error, heading error, and vertical velocity error. These error scores were computed using the following formula:

$$
\mathrm{E}=\mathrm{SD}+(\mathrm{ABS}(\mathrm{X}-\mathrm{A}) / \mathrm{W})^{2} \text {, }
$$

where $\mathrm{E}=$ error score, $\mathrm{SD}=$ standard deviation, $\mathrm{ABS}$ $=$ absolute value, $\mathrm{X}=$ measured value, $\mathrm{A}=$ assigned value, and $\mathrm{W}=$ "window" value. Basically, the error score consisted of the standard deviation weighted by the number of "window" units that the mean differed from the assigned value (for that maneuver), with the weighted quantity squared. For example, if the pilot flew an average heading in an SLT that was $5^{\circ}$ off the assigned heading, the error score would be the standard deviation of the 600 heading samples taken during the maneuver plus a weighting factor of +4 (the $5^{\circ}$ heading error is twice the "window" criterion of $\pm 2.5^{\circ}$, which is then,squared to give a value of 4). The weighting factor was based on the theory that pilots tend to fly using a window-type control criterion in which they do not usually attempt to correct errors until they get outside the window value (Perelli, 1980). The penalty in the weighting is minimal for values inside the window, but increases exponentially for values outside the window. This penalty structure was based on a study by De Greene (1970), in which he stated, "It may be hypothesized that pilots control their craft in such a way as to minimize the mean square tracking errors" (p. 204).

After the error scores were computed, they were converted to $Z$-score values (within subject) and averaged across all four flight parameters to yield a single score for each pilot for each SLT or FMT (16 error scores for each pilot).

The performance scores calculated by the "window" method (Perelli, 1980), called "combined total seconds of error (CTSE)," were converted to proportions representing the ratio of total number of seconds of error to total possible number of seconds of error. These proportional values were then subjected to arcsine transformation (Cohen \& Cohen, 1975) so that they could be averaged across maneuvers to yield a single score for each flight task. The transformed scores were then converted to Z-score equivalents to allow comparison with the " $E$ " values discussed above.

The eye movement data consisted of two channels (vertical eye movement and horizontal eye movement) recorded on analog tape. The tape was digitized at $100 \mathrm{~Hz}$ into groups of digital data. Each group represented one SLT or FMT for a given subject. The digitized eye movement data were processed using the ATDEYE program adapted from a FORTRAN program furnished by J. Stern and L. Walrath, Washington University Behavior Research Laboratory, St. Louis, Missouri. The output of this program gave a summary for each minute of each maneuver. The summary contained means, standard deviations, and frequency counts for eyeblinks and horizontal saccades. These data were then reduced to a single summary for each SLT or FMT consisting of Z-score values for each of the applicable parameters from the EOG.

The final data were set up as a Statistical Analysis System (SAS) data file. This file consisted of one Z-score value for each variable for each of 10 subjects for each SLT and FMT.

\section{Analyses and Experimental Design}

Due to a limited availability of subjects and the difficult technical nature of this investigation, the subject sample size was small. Repeated measures were necessary due to the use of fatigue as a stressor. The statistical power of this type of design is generally rather low; however, this investigation was very task specific in nature, and the subjects were highly representative of the small population to which data were extrapolated. The criterion for a significant change in performance was one standard deviation. Using this value for population "effect" (gamma) and selecting the 0.95 level of confidence required a minimum sample size of eight to have a statistical power ( 1 -Beta) of 0.80 (Welkowitz, Ewen, \& Cohen, 1973). The independent variable was the "fatigue" stress associated with time on task. However, the data analyses of interest were the correlations between two variables thought to be highly sensitive to time on task. These variables were the performance measures and the corresponding EOG measures. Eight epochs for each of the two performance tasks (SLTs and FMTs) were scored, with concurrent EOG data, for each of the 10 subjects, giving 160 data points.

\section{RESULTS}

All data were plotted in Z-score equivalents in order that dissimilarly scaled variables could be graphed 
together. All data analyses were performed on the Data Sciences Division's VAX 11/780 system using SAS (Barr, Goodnight, Sall, Blair, \& Chilco, 1979).

\section{Performance}

The first step was to examine the a priori assumption that performance would be significantly degraded over time. Plotting the change in error score (E) over the eight epochs (legs) of the sortie showed that error increased until the last segment (Epoch 8) and then decreased slightly. A one-way analysis of variance (ANOVA) on $E$ (Table 1) indicated a significant change $[F(7,152)=16.58, p<$ $.0001]$ across the eight epochs. A follow-up analysis using Duncan's multiple range test showed differences between the early, middle, and late periods of the sortie.

An ANOVA of the change in the accuracy-based performance measure, CTSE over time, was not significant $[F(7,152)=0.35, p<.93]$. Since no significant change occurred in the CTSE measure over time, it was not included in any further analyses of the data.

\section{Eye Movement Measures}

The Pearson product moment correlation (Table 2) was calculated for the relationships between error $(E)$ and each of the eye movement (EOG) measures.

Two of the three blink parameters, blink duration (BD) and blink amplitude (BA), yielded moderately high correlations, whereas blink rate (BR) was low but significant. Long closure rate $(\mathrm{LCR}=$ the frequency of eye closures greater than $500 \mathrm{msec}$ ) yielded a moderaterly high correlation. And one saccade measure, saccade rate (SR), yielded a moderately high correlation, whereas the other two, mean saccade velocity (SV) and peak saccade velocity (PSV), were not significantly correlated.

It was determined that the two types of flying tasks in the sortie (FMTs and SLTs) were different in type. Accordingly, the relationships between EOG and E score were examined for the combined data set, as well as for FMTs and SLTs separately.

Combined data analysis. To complete the examination of the combined data set (FMTs and SLTs), a stepwise regression analysis was performed to determine to what degree EOG measures were predictors of performance based on the $\mathrm{E}$ score. The F-test significance value was set to $p<.05$ for any single variable to enter the model. The best single fit was the variable $\mathrm{BA}[\mathrm{F}(1,158)$ $=88.27, \mathrm{p}<.0001]$ with $\mathrm{R}$ squared equal to 0.36 . The best two-variable fit was with LCR entered as the second

Table 1

Analysis of Variance for Error (E)

\begin{tabular}{lrrrrr}
\hline \multicolumn{1}{c}{ Source } & DF & SS & MS & F & p \\
\hline Model & 7 & 24.5848 & 3.5121 & $16.58^{*}$ & $.0001^{*}$ \\
Error & 152 & 32.1905 & .2118 & & \\
Total (C) & 159 & 56.7753 & & & \\
R squared $=0.43$ & & & & \\
\hline
\end{tabular}

*Significant value.
Table 2

Correlation Table for Performance and EOG Measures

\begin{tabular}{cccccccc}
\hline Variable & BR & BD & LCR & SV & BA & PSV & SR \\
\hline \multirow{2}{*}{ E } & $0.164^{*}$ & $0.496^{*}$ & $0.562^{*}$ & 0.041 & $-0.599^{*}$ & 0.027 & $-0.472^{*}$ \\
& 0.0378 & 0.0001 & 0.0001 & 0.6052 & 0.0001 & 0.7374 & 0.0001 \\
\hline
\end{tabular}

Note $-N=160$ (data points). Upper number is Pearson product moment correlation coefficient. Lower number is level of significance. $E$ $=$ error score (variability based); $B R=$ blink rate; $B D=$ blink duration; $L C R=$ long closure rate; $S V=$ saccade velocity; $B A=$ blink amplitude; $P S V=$ peak saccade velocity; $S R=$ saccade rate. *Significant value.

variable $[F(2,157)=92.24, p<.0001]$ with $R$ squared equal to 0.54 . The best three-variable fit was with $\mathrm{BD}$ as the third variable $[\mathrm{F}(3,156)=81.48, \mathrm{p}<.0001]$ with $\mathrm{R}$ squared equal to 0.61 .

Analysis of FMTs only. The stepwise regression showed $B A$ to be the best single variable $[F(1,78)=26.1$, $p<.0001], B A$ and $B R$ the best two variables $[F(2,77)$ $=36.12, \mathrm{p}<.0001 \mathrm{]}, \mathrm{BA}, \mathrm{BR}$, and LCR the best three variables $[F(3,76)=35.31, p<.0001]$, and $B A, B R$, LCR, and SR the best four variables $[F(4,75)=33.15$, $\mathrm{p}<.0001]$ with $R$ squared values of $0.25,0.48,0.58$, and 0.64 , respectively.

Analysis of SLTs only. The stepwise regression indicated that the best single predictor was LCR $[F(1,78)=$ $53.96, p<.0001]$ and the best second variable with LCR was $\mathrm{BA}[\mathrm{F}(2,77)=70.41, \mathrm{p}<.0001]$. $\mathrm{R}$ squared values for the one- and two-variable models were 0.41 and 0.65 , respectively.

\section{Summary of Results}

The general results of all data analyses are as follows:

(1) Error score (E) increased significantly over time.

(2) Combined total seconds of error (CTSE) did not change significantly over time.

(3) For the combined data set, blink amplitude (BA) was the best predictor of changes in $E$, with decreased amplitude corresponding to increased $E$. Peak saccade velocity (PSV) was not a significant predictor.

(4) For FMTs only, BA was the best predictor of E, with decreased amplitude corresponding to increased $E$. BR and LCR were the second- and third-best predictors, respectively. PSV was not a significant predictor.

(5) For SLTs only, LCR and BA were the first- and second-best predictors of increased E, respectively. PSV was not a signifiant predictor.

\section{DISCUSSION}

The most significant outcome of this study is the development of a performance-evaluation system, using a flight simulator, which allows the study of physiological correlates to stress effects. The E score appears to be a viable performance measure for this type of complex manual control task in which relatively continuous samples of position are obtained. Comparison of the $\mathrm{E}$ score with the previously used "window" scoring methods has 
demonstrated that the E score is more sensitive to the subtle changes in variability of performance due to fatigue.

The results of the data analyses indicate that the EOG is a viable measure with which to monitor the effects of fatigue and other stressors on pilots. The measures best reflecting this relationship are blink amplitude, long closure rate, blink rate, and blink duration. However, no single variable accounts for more than $41 \%$ of the variability in the regression. This suggests that some combined metric based on the change in all four EOG measures may be the best application. Follow-up studies are under way to evaluate the utility of these measures in the operational environment.

Future development of the system includes installation of analog-to-digital capability onboard the simulator. This would allow digital data to be transmitted to the computer for analysis and thereby reduce the problem of noise induced into the data link. This would also eliminate the need for separate digitization of the EOG data before analysis with the ATDEYE program.

Future extension of the data obtained from this study and other, similar data might lead to development of a biocybernetic system in which a continuous physiological monitoring of the pilot could provide immediate feedback to increase pilot arousal levels. Such a system might also activate an automated device to take control when the pilot is no longer capable of safely operating an aircraft.

\section{REFERENCES}

Barr, A. J., Goodnight, J. H., Sall, J. P., Blair, W. H., \& Chilco, D. M. (1979). SAS user's guide. Raleigh, NC: SAS Insitute, Inc. Beideman, L. R., \& STERN, J. A. (1977). Aspects of the eyeblink during simulated driving as a function of alcohol. Human Factors, 19 , 73-77.

BERGUM, B. O., \& LEHR, D. J. (1962). Vigilance performance as a function of interpolated rest. Journal of Applied Psychology, 46, 425-430.
Cohen, J., \& CoHEN, P. (1975). Applied multiple regression/correlation analysis for the behavioral sciences. New York: Wiley.

Colquhoun, W. P. (1959). The effect of a short rest pause on inspection efficiency. Ergonomics, 2, 367-376.

DE GREene, K. B. (1970), Systems psychology. New York: McGraw-Hill.

Henry, P. H., Davis, T. Q., Engelken, E. J., Triebwasser, J. H., * Lancaster, M. E. (1974). Alcohol-induced performance decrement assessed by two Link trainer tasks using experienced pilots. Aerospace Medicine, 45, 1180-1189.

Henry, P. H., Flueck, J. A., SANFord, J. F., Keiser, H. N., MCNeE, R. C., Walter, W. H., III, Webster, K. H., Hartman, B. O., \& LANCASTER, M. C. (1974). Assessment of performance in a Link GAT-1 flight simulator at three alcohol dose levels. Aerospace Medicine, 45, 33-44.

Henry, P. H., Turner, R. A., \& Matthie, R. B. (1974). An automated system to assess pilot performance in a Link GAT-1 trainer (Tech. Rep. No. 79-41). Brooks Air Force Base, TX: USAF School of Aerospace Medicine, Aerospace Medical Division.

JAHNS, D. W. (1973, December). A concept of operator workload in manual vehicle operations. Paper presented at the NATO Defense Resarch Group Seminar on Man-Machine Relations, Utrecht, The Netherlands.

MutTo, W. H., \& WierWILLE, W. W. (1982). The effect of repeated emergency response task on performance during extended-duration simulated driving. Human Factors, 24, 693-698.

OSTER, P. J., \& STERN, J. A. (1980). Measurement of eye movement In I. Martin \& P. H. Venables (Eds.), Techniques in psychophysiology. New York: Wiley.

PERELLI, L. P. (1980). Fatigue stressors in simulated long-duration flight: Effects on performance, information processing, subjective fatigue, and physiological cost (Tech. Rep. No. 80-49). Brooks Air Force Base, TX: USAF School of Aerospace Medicine, Aerospace Medical Division.

Stave, A. M. (1977). The effects of cockpit environment on long-term pilot performance. Human Factors, 19, 503-514.

Sussman, D. (1970). An investigation of factors affecting driver alertness (Tech. Rep. No. FH-11-7313). Buffalo, NY: Cornell Aeronautical Laboratories.

Threatt, D. H., \& Perelli, L. P. (1977). Computer program listings, flight plans and data files for the GAT-1 flying performance and physiological measurement system. Brooks Air Force, Base, TX: USAF School of Aerospace Medicine, Aerospace Medical Division. WeLkowtTZ, J., EWEN, R. B., \& CoHEN, J. (1973). Introductory statistics for the behavioral sciences. New York: Academic Press. 